\title{
Coexistence of granulomatosis with polyangiitis (GPA) and Crohn's disease or multiorgan manifestation of the same disease?
}

\author{
Lucyna Jóźwiak, Izabela Ławnicka, Andrzej Książek \\ Department of Nephrology, Medical University of Lublin, Lublin, Poland
}

\begin{abstract}
Granulomatosis with polyangiitis (GPA) is a systemic necrotizing vasculitis of unknown aetiology, often related to the antineutrophil cytoplasmic antibody (ANCA). GPA was previously named Wegener's granulomatosis (WG). The disease frequently has multisystemic presentation, targeting mainly the respiratory tract and kidneys, but gastrointestinal involvement is uncommon. Crohn's disease $(C D)$ is an inflammatory bowel disease (IBD) with many extraintestinal manifestations. Clinically, symptoms of WG and CD can mimic each other. In this paper a case of GPA manifested initially by severe multiorgan damage including colitis, regarded to be coexistent $C D$, is presented. The case illustrates the difficulties in establishing the diagnosis when symptoms of the diseases mimic each other.
\end{abstract}

Key words: granulomatosis with polyangitis, ANCA antibody, Crohn's disease.

\section{Introduction}

Granulomatosis with polyangiitis (GPA) is a systemic necrotizing vasculitis of unknown aetiology, which affects small and medium vessels often related to anti-neutrophil cytoplasmic antibody (ANCA) and most commonly affects the upper respiratory tract, lungs and kidney.

GPA was previously named Wegener's granulomatosis (WG), but according to the Chapel Hill Consensus Conference Nomenclature [1] WG is currently named granulomatosis with polyangiitis (GPA).

The clinical course of the disease frequently has multisystemic presentation. Based on the clinical picture five forms of GPA are differenced: limited, early systemic, generalized, severe generalized and resistant to treatment form [2].

The common general symptoms of GPA are: fatigue, arthralgia, fever, skin abnormalities and weight loss [3]. It very seldom involves gastrointestinal organs $[4,5]$. Crohn's disease is an inflammatory bowel disease that may be associated with a variety of extraintestinal man- ifestations [6]. The respiratory tract is less often affected compared with other organs [7].

Clinically, symptoms of GPA and Crohn's disease can mimic each other.

We present a case of GPA manifested initially by severe multiorgan damage including colitis, regarded to be coexistent Crohn's disease.

\section{Case report}

A 54-year-old man, previously healthy, was admitted to the hospital with fever, joint pain, generalized swelling, weakness, weight loss, anaemia, abdominal pain, bloody diarrhoea and maculopapular rash of his limbs (Figs. 1, 2).

Laboratory studies revealed high inflammation markers (C-reactive protein [CRP] $269 \mathrm{mg} / \mathrm{dl}$, procalcitonin $18.44 \mathrm{ng} / \mathrm{ml}$, leukocytosis - WBC $13.4 \mathrm{~K} / \mathrm{l}$ ), anaemia (Hgb $8.9 \mathrm{~g} / \mathrm{dl})$, elevated levels of fibrinogen $(5.7 \mathrm{mg} / \mathrm{dl})$ and D-dimer $(8.115 \mathrm{~g} / \mathrm{ml})$, mildly impaired renal function (creatinine $1.3 \mathrm{mg} / \mathrm{dl}$, eGFR $60 \mathrm{ml} / \mathrm{min} / 1.73 \mathrm{~m}^{2}$ ) and nephritic urine sediment. Proteinuria did not exceed $3.0 \mathrm{~g} / 24 \mathrm{~h}$.

\footnotetext{
Address for correspondence:

Lucyna Jóźwiak, Department of Nephrology, Medical University of Lublin, Jaczewskiego 8, 20-950 Lublin, Poland,

e-mail: lucyna.jozwiak@interia.pl

Submitted: 14.03.2016; Accepted: 25.04.2016
} 


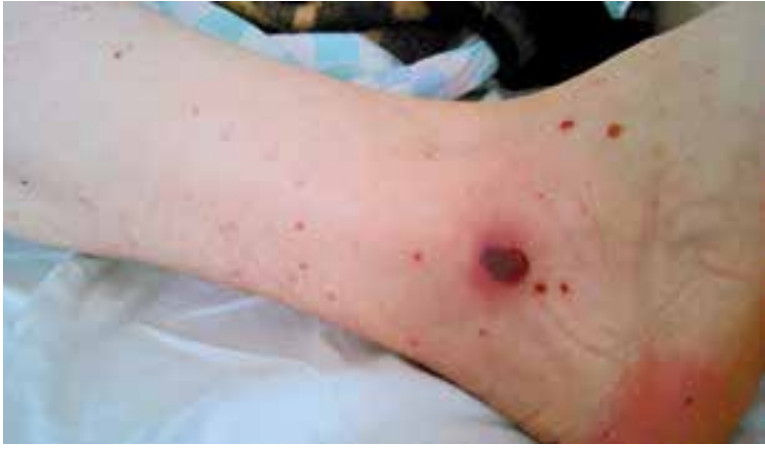

Fig. 1. Maculopapular rash of the lower limb.

The levels of immunoglobulin (IgG, IgA, IgM, IgE) and C3 and C4 components of complement remained normal.

Blood and urine culture, stool examination for the presence of rotavirus, adenovirus and Salmonella/Shigella were negative. No toxins A and B for Clostridium difficile were detected.

In the following days, the condition of the patient deteriorated. He required constant catecholamine infusion. The abdomen was distended, and bloody diarrhoea persisted. The plain abdominal X-ray showed fluid levels. The abdominal ultrasound showed homogeneous liver, spleen, and kidney enlargement, decreased motility of the small intestine, features of subileus, thickened walls of the small intestine, and free fluid in the rectovesical excavation.

In the diagnostic colonoscopy, the ileum was inflamed, swollen, with small ulcers and inflammatory polyps. The Bauhin valve was also inflamed and swollen, and numerous deep and longitudinal ulcers in the area of the ascending colon were found. Crohn's disease was suspected. The histopathological examination of collected clippings found equivocal features of chronic inflammatory bowel disease, without suggestion of blood vessel inflammation typical for GPA.

Treatment with mesalazine (total daily dose 4500 mg) was immediately started.

In addition chest $\mathrm{X}$-ray showed bilateral increased bronchial patterns of the lungs and bilateral peribronchial patchy consolidations.

For further evaluation intravenous contrast-enhanced chest CT was performed. It revealed infiltrations and nodules up to $9 \mathrm{~mm}$ (Fig. 3) in both lungs; the infiltrates measured approximately $29 \mathrm{~mm}$ in diameter (Fig. 4) in the apex of the right lung.

The whole clinical picture suggested systemic vasculitis. The diagnosis was confirmed after receiving the result of the ANCA antibodies. The level of antibodies against proteinase 3 (c-ANCA, PR-3) was greater than $150 \cup(N<20)$. Further dilution was not performed. The

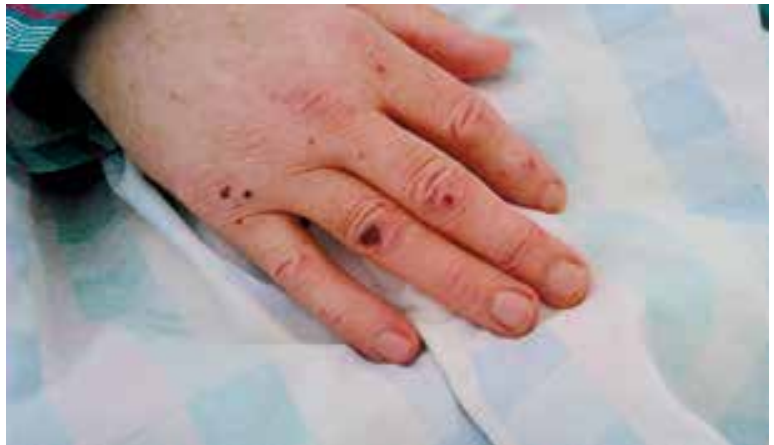

Fig. 2. Maculopapular rash of the palm.

level of antibodies against myeloperoxidase ( $p$-ANCA, MPO) was normal. No antinuclear antibodies (ANA) or anti-glomerular basement membrane (anti-GBM) antibodies were detected. Rheumatoid factor (RF) was at the upper limit of normal $-30 \mathrm{U} / \mathrm{ml}(\mathrm{N}<30 \mathrm{U} / \mathrm{ml})$.

The patient was diagnosed with severe granulomatosis with polyangiitis (GPA) and coexistent Crohn's disease. Immunosuppressive therapy was started very cautiously with an infusion of methylprednisolone with protection of broad spectrum antibiotics because of the constant septic state. The patient received in total 3000 mg of methylprednisolone, with continuation of orally taken prednisone. Infusions of cyclophosphamide (CF) at a dose of about $7.5 \mathrm{mg} / \mathrm{kg}$ and the infusion of immunoglobulins were added to the current therapy. Inflammatory bowel disease was treated with orally taken mesalazine. Throughout the 2 months of hospitalization, the patient remained anaemic, requiring packed red blood cell transfusions. Diagnostic biopsy of the kidney was not done because of severe heart failure during the first two weeks of hospitalization.

As a result of the treatment, the patient's overall condition improved, allowing for bronchofibroscopy, which showed inflammatory infiltrations in the course of systemic vasculitis and severe pneumonia. In the sputum multiple resistant strains of Acinetobacter baumannii complex were cultured, sensitive only to colistin. A targeted antibiotic was ordered. The patient was then treated with monthly infusions of cyclophosphamide and orally prednisone. His condition improved considerably. Radiographic changes in the lungs almost completely resolved. C-reactive protein (CRP) and procalcitonin normalized. Colonoscopy was performed 11 months later and showed no abnormalities.

The total dose of cyclophosphamide received by the described patient was $7 \mathrm{~g}$, which is equivalent to $10 \mathrm{mg} / \mathrm{kg}$ of body weight. The patient continues treatment with a maintenance dose of prednisone $5 \mathrm{mg} /$ day. 


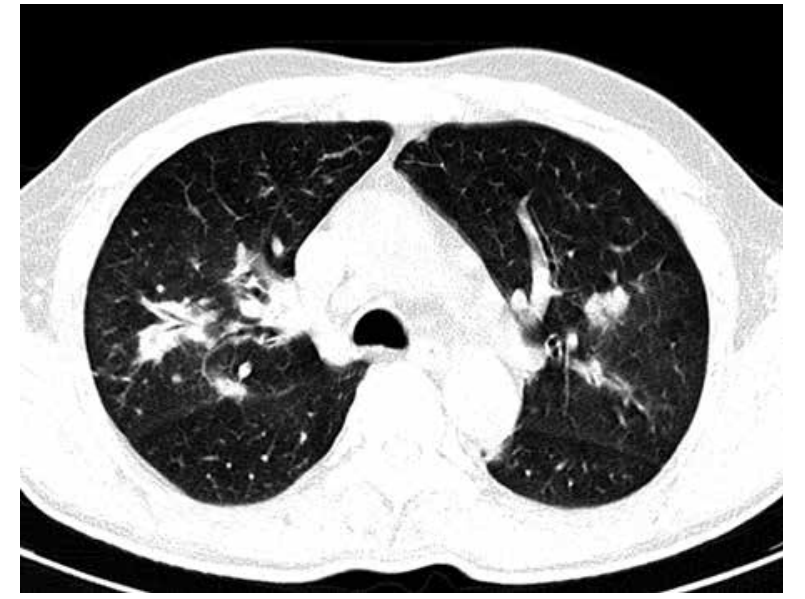

Fig. 3. Intravenous contrast-enhanced chest CT image: infiltrates and nodules of both lungs.

\section{Discussion}

Coexistence of ANCA-associated vasculitis (AAV) and inflammatory bowel disease (IBD) is rarely described in the literature.

Granulomatosis with polyangiitis (GPA) can present with symptoms covering multiple systems [3, 8], but most commonly involves the upper and lower respiratory tract and the kidneys. Clinically significant involvement of the gastrointestinal system is infrequent, usually after several years of GPA and its treatment [4].

The present patient was admitted to the hospital because of various nonspecific symptoms: fatigue, weight loss, arthralgia, anaemia, rash, oedema, abdominal pain, fever and bloody diarrhoea. The search for infectious causes remained negative, so colonoscopy was performed and revealed inflammatory and ulcerative lesions implying Crohn's disease, but histopathological features were equivocal. In this case a diagnostic possibility was intestinal involvement in GPA or coexistence of GPA and Crohn's disease. We did not believe Crohn's disease to be the sole cause of multi-organ symptoms in the patient, because in Crohn's disease overt lung involvement is rare [9] whereas CT of the lung showed severe abnormalities.

Gastrointestinal involvement has been described in $10-24 \%$ of patients with GPA $[10,11]$. In the majority of the reports, the colitis developed up to several years after GPA diagnosis and treatment. There are also cases of patients with Crohn's disease who later developed GPA [12]. In 2010 the first known example of hemorrhagic colitis as an initial presentation of GPA was described [13], and since then GPA should be considered in the differential diagnosis of colitis of unclear aetiology.

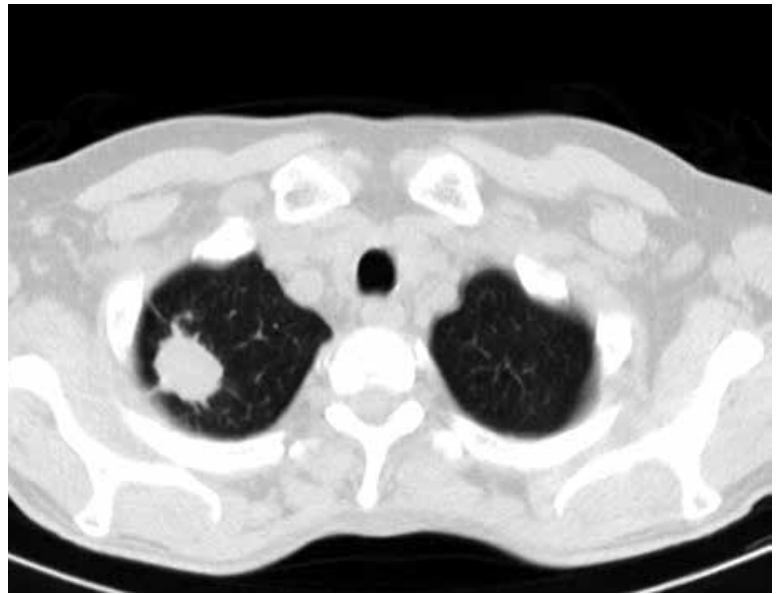

Fig. 4. Intravenous contrast-enhanced chest CT image: infiltration in the apex of the right lung.

Crohn's disease (CD) is an inflammatory bowel disease that can involve various organ systems and presents with many extraintestinal manifestations mimicking common symptoms of GPA such as arthralgia, fever, skin abnormalities, and weight loss.

In 2015 in a retrospective study seven patients with granulomatosis with polyangiitis (GPA) and Crohn's disease were described [14] but only in two cases did IBD start simultaneously with AAV manifestations. The present patient had a typical clinical presentation of CD, and colonoscopy showed characteristic abnormalities but without clear confirmation by histological examination.

Crohn's disease and GPA are both associated with ANCA [15], but show a distinctive distribution. Cytoplasmic staining ANCA (c-ANCA) reacting with proteinase 3 (PR-3) predominates in GPA and may be present in Crohn's disease, whereas perinuclear ANCA ( $p$-ANCA) reacting with a myeloperoxidase, elastase, lactoferrin, cathepsin $G$ and other neutrophil antigens have been reported in inflammatory bowel diseases, mainly in ulcerative colitis (50-80\%) and in Crohn's disease (1040\%) [16].

Crohn's disease and GPA are also associated by common pathogenic mechanisms. Both diseases are considered granulomatous Th1-mediated diseases. In GPA and in Crohn's disease the key role of Th1 and Th17 lymphocytes is known $[17,18]$. It is currently known that anti-Saccharomyces cerevisiae antibodies (ASCAs) are more frequently found in Crohn's disease and are a good biomarker of this disease [19], but they have not been evaluated in patients described.

In the present patient c-ANCAs in a very high titre were detected. 
Renal disease in GPA mostly manifests as microscopic haematuria, proteinuria and an elevated serum creatinine concentration. The reported patient presented all above features.

The patient was diagnosed with GPA on the basis of the clinical presentation: the lower respiratory tract infiltrates that are characteristic for the disease, typical renal involvement and the presence of c-ANCAs in a high titre that are strongly associated with GPA. The chest $X$-rays and $\mathrm{CT}$ of the lungs that showed nodular lesions and migratory infiltrates were characteristic for GPA, although the clinical picture was distorted by severe pulmonary infection. Gastrointestinal abnormalities manifesting as abdominal pain, weight loss, and bloody diarrhoea with mucus could have been considered as clinical features of GPA since intestinal involvement of GPA mimicking Crohn's disease has been described [20]. The temporal association with pulmonary and kidney manifestations and the responsiveness to the immunosuppressive drugs support colitis as a manifestation of GPA. On the other hand, coexistence of two diseases was also possible because characteristic features of inflammatory bowel disease were found in colonoscopy. Because the results of the colonoscopy were known prior to the ANCA tests, mesalazine as the treatment of IBD was implemented and has been continued together with immunosuppressants added after c-ANCA detection. So the treatment was targeted at GPA as well as at Crohn's disease.

The patient responded well to a combination therapy of steroids, cyclophosphamide and mesalazine with resolution of intestinal and pulmonary symptoms. The kidney function remained slightly impaired.

The present case illustrates the difficulties of establishing the diagnosis when symptoms of the diseases mimic each other. Problems with differentiation can also result from an atypical course of GPA [21].

$$
* * *
$$

In conclusion, the present case is one of the very few cases of coexisting GPA and CD simultaneously diagnosed described in the English language literature.

The authors declare no conflict of interest.

\section{References}

1. Jennette JC, Falk RJ, Bacon PA, et al. 2012 revised International Chapel Hill Consensus Conference Nomenclature of Vasculitides. Arthritis Rheum 2013; 65: 1-11.

2. Madej M, Matuszewska A, Białowąs K, et al. Clinical forms of granulomatosis with polyangiitis. Reumatologia 2014; 52: 332-338.
3. Hoffman GS, Kerr GS, Leavitt RY, et al. Wegener granulomatosis: an analysis of 158 patients. Ann Intern Med 1992; 116: 488-498.

4. Storesund B, Gran JT, Koldingsnes W. Severe intestinal involvement in Wegener's granulomatosis: report of two cases and review of the literature. Br J Rheumatol 1998; 37: 387-390.

5. Hawoth SJ, Pusey CD. Severe intestinal involvement in Wegener's granulomatosis. Gut 1984; 25: 1296-1300.

6. Levine JB, Lukawski-Trubish D. Extraintestinal consideration in inflammatory bowel disease. Gastroenterol Clin North Am 1995; 24: 633-646.

7. Camus P, Piard F, Ashcroft T, et al. The lung in inflammatory bowel disease. Medicine (Baltimore). 1993; 72: 151-183.

8. Fauci AS, Haynes BF, Katz P, et al. WG: prospective clinical and therapeutic experience 85 patients for 21 years. Ann Intern Med 1983; 98: 76-85.

9. Casey MB, Tazelaar HD, Myers JL, et al. Noninfectious lung pathology in patients with Crohn's disease. Am J Surg Pathol 2003; 27: 213-219.

10. Izzedine H, Lacaille S, Deray G. An unusual presentation of relapsing Wegener's granulomatosis. Nehrol Dial Transplant 2001; 16: 1511-1512.

11. Deniz K, Ozseker HS, Balas S, et al. Intestinal involvement in Wegener's granulomatosis. J Gastrointesin Liver Dis 2007; 16: 329-331.

12. Sieczkowska A, Landowski P, Szumera M, et al. Coexistance of Crohn disease and Wegener granulomatosis in a 15-year-old patient. Med Wieku Rozwoj 2011; 15: 472-476.

13. Qian Q, Cornell L, Chandan V, et al. Hemorrhagic colitis as a presenting feature of Wegener granulomatosis. J Gastrointestin Liver Dis 2010; 19: 445-447.

14. Humbert S, Guilpain P, Puechl X, et al. Inflammatory bowel diseases in anti-neutrophil cytoplasmic antibody-associated vasculitides: 11 retrospective cases from the French Vasculitis Study Group. Rheumatology (Oxford) 2015; 54: 1970-1975.

15. Kallenberg CG, Brouwer E, Weening JJ, et al. Anti-neutrophil cytoplasmic antibodies: current diagnostic and pathophysiological potential. Kidney Int 1994; 46: 1-15.

16. Rao JK, Weinberger M, Oddone EZ, et al. The role of antineutrophil cytoplasmic antibody (c-ANCA) testing in the diagnosis of Wegener's granulomatosis. Ann Intern Med 1995; 113: 925-932.

17. Csernok E, Trabandt A, Muller A, et al. Cytokine profiles in Wegener's granulomatosis: predominance of type 1 (Th1) in the granulomatous inflammation. Arthritis Rheum 1999; 42: 742-750.

18. Fuss IJ, Becker C, Yang Z, et al. Both IL -12 p70 and IL-23 are synthesized during active Crohn's disease and are down-regulated by treatment with ani-IL-12 p40 monoclonal antibody. Inflam Bowel Dis 2006; 12: 9-15.

19. Desplat-Jégo S, Johanet C, Escande A, et al. Update on antiSaccharomyces cerevisiae antibodies, anti-nuclear associated anti-neutrophil antibodies and antibodies to exocrine pancreas detected by indirect immunofluorescence as biomarkers in chronic inflammatory bowel diseases: results of a multicenter study. World J Gastroenterol 2007; 13: 23122318. 
20. Sokol RJ, Farrell MK, McAdams AJ. An unusual presentation of Wegener's granulomatosis mimicking inflammatory bowel disease. Gastroenterology 1984; 87: 426-432.

21. Madej M, Matuszewska A, Białowąs K, et al. Ziarniniakowatość z zapaleniem naczyń - problemy diagnostyczne. Reumatologia 2014; 52: 344-346. 